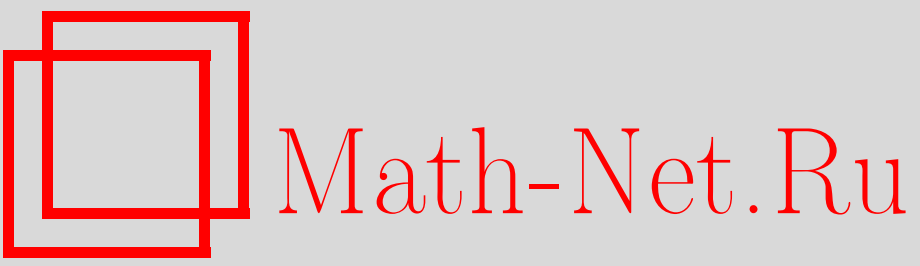

Ю. П. Соловьев, В. В. Белокуров, Е. Т. Шавгулидзе, Существование функциональных интегралов в квантовополевой модели на пространстве петель, $У M H$, 2004, том 59, выпуск 5, 163-164

DOI: https://doi.org/10.4213/rm785

Использование Общероссийского математического портала Math-Net.Ru подразумевает, что вы прочитали и согласны с пользовательским соглашением

http: //www . mathnet.ru/rus/agreement

Параметры загрузки:

IP : 54.198 .67 .100

26 апреля 2023 г., 12:39:10 


\title{
СУЩЕСТВОВАНИЕ ФУНКЦИОНАЛЬНЫХ ИНТЕГРАЛОВ В КВАНТОВОПОЛЕВОЙ МОДЕЛИ НА ПРОСТРАНСТВЕ ПЕТЕЛЬ
}

\author{
Ю. П. СОлОВьЕВ, В. В. БЕлОКУРов, Е. Т. ШАВГУлИДЗЕ
}

На пространстве петель построим обобщение квантовой теории поля следующим образом. В качестве импульсного пространства выберем $\mathscr{P}=C\left(S^{1}, \mathbb{R}^{4}\right)$ - банахово пространство всех непрерывных отображений окружности единичной длины в $\mathbb{R}^{4}$ с нормой $\|p\|_{\mathscr{P}}=\max _{\tau \in S^{1}}\|p(\tau)\|$, где $\|\cdot\|$ - каноническая норма в пространстве $\mathbb{R}^{4}$.

На пространстве $\mathscr{P}$ задано представление группы $G=\operatorname{Diff}_{+}^{3}\left(S^{1}\right)$ трижды непрерьвно диффференцируемых диффеоморфизмов $g \in G\left\{g: S^{1} \rightarrow S^{1}, g^{\prime}(\tau)>0\right\}$.

Представим пространство $\mathscr{P}$ в виде декартова произведения $\mathscr{P}=\mathbb{R}^{4} \times \mathscr{P}_{0}$, где $\mathscr{P}_{0}=$ $\{p \in \mathscr{P}: p(0)=0\}, p(\tau)=r+\xi(\tau), p \in \mathscr{P}, r \in \mathbb{R}^{4}, \xi \in \mathscr{P}_{0}$.

Зададим на $\mathbb{R}^{4}$ меру Лебега $l$ и на $\mathscr{P}_{0}$ броуновский мост $w_{\lambda}^{0}$ с коэффициентом дисперсии $\sqrt{\lambda}$, т.е. условную меру Винера $(p(0)=p(1)=0)$ на отрезке $[0,1]$ (полуинтервал $[0,1)$ отождествляется с окружностью $\left.S^{1}\right)$.

Тем самым на пространстве $\mathscr{P}$ задана мера Винера $w_{\lambda}=l \otimes w_{\lambda}^{0}$.

Обозначим через $E$ пространство всех квадратично интегрируемых по мере Винера функций $\varphi: \mathscr{P} \rightarrow \mathbb{C}$ таких, что $\varphi(p)=\overline{\varphi(-p)}$ для любых $p \in \mathscr{P}$.

Пространство $E$ является гилшбертовым пространством над вещественным полем $\mathbb{R}$ со скалярным произведением $(\varphi, \phi)_{E}=\int_{\mathscr{P}} \varphi(p) \overline{\phi(p)} w_{\lambda}(d p)$.

Функции $\varphi$ и $\phi$ реализуют регулярное унитарное представление группы дифффеоморфизмов $G$ в гильбертовом пространстве $E$.

Зададим свободное действие, которое инвариантно относительно группы $G$ :

$$
\mathscr{A}_{0}[\varphi]=\int_{\mathscr{P}}|\varphi(p)|^{2} \Omega^{2}(p) w_{\lambda}(d p), \quad \Omega^{2}(p) \equiv\left(\int_{S^{1}} \frac{d \tau}{\|p(\tau)\|^{2}}\right)^{-1}+m^{2} .
$$

В пределе оно переходит в свободное действие скалярного поля,

$$
\lim _{\lambda \rightarrow+\infty} \mathscr{A}_{0}[\varphi]=\int_{\mathbb{R}^{4}}|\varphi(r)|^{2}\left(\|r\|^{2}+m^{2}\right) d r .
$$

Взаимодействие, в локальном пределе переходящее в $\varphi^{4}$, может быть записано в виде

$$
\begin{aligned}
& \mathscr{A}_{1}[\varphi]=\int_{\mathscr{P}} \ldots \int_{\mathscr{P}} \varphi\left(p_{1}\right) \mathscr{U}_{\alpha \lambda}\left(p_{1}\right) \Delta\left(p_{1}, p_{5}\right) \varphi\left(p_{2}\right) \mathscr{U}_{\alpha \lambda}\left(p_{2}\right) \Delta\left(p_{2}, p_{6}\right) \varphi\left(p_{3}\right) \mathscr{U}_{\alpha \lambda}\left(p_{3}\right) \Delta\left(p_{3}, p_{7}\right) \\
& \quad \times \varphi\left(p_{4}\right) \mathscr{U}_{\alpha \lambda}\left(p_{4}\right) \Delta\left(p_{4}, p_{8}\right) \Delta\left(p_{5},-p_{6}-p_{7}-p_{8}\right) \\
& \quad \times \mathscr{U}_{\alpha \lambda}\left(p_{5}\right) \mathscr{U}_{\alpha \lambda}\left(p_{6}\right) \mathscr{U}_{\alpha \lambda}\left(p_{7}\right) \mathscr{U}_{\alpha \lambda}\left(p_{8}\right) w_{\lambda}\left(d p_{1}\right) \cdots w_{\lambda}\left(d p_{8}\right) .
\end{aligned}
$$

Здесь введены обозначения

$$
\begin{aligned}
\Delta\left(p_{1}, p_{5}\right) & \equiv \int_{S^{1}} \frac{d \tau}{\left\|p_{1}(\tau)\right\|^{2}} \int_{S^{1}} \delta\left(p_{1}\left(\tau_{1}\right)-p_{5}\left(\tau_{1}\right)\right)\left\|p_{1}\left(\tau_{1}\right)\right\|^{2} d \tau_{1}, \\
\mathscr{U}_{\alpha \lambda}(p) & \equiv \int_{G} \exp \left\{\frac{\lambda}{8} \int_{S^{1}} \mathscr{S}_{g}(\tau)\|p(\tau)\|^{2} d \tau\right\} \mu_{\alpha \lambda}(d g),
\end{aligned}
$$

где

$$
\mathscr{S}_{g}=\frac{g^{\prime \prime \prime}(\tau)}{g^{\prime}(\tau)}-\frac{3}{2}\left(\frac{g^{\prime \prime}(\tau)}{g^{\prime}(\tau)}\right)^{2}
$$

Работа выполнена при поддержке Российского фонда фундаментальный исследований (грант № 03-01-00371). 
- шварциан и $\mu_{\alpha \lambda}$ - мера на группе $G$, которая строится следующим образом [1]. Возьмем подгруппу $G_{0}=\{g \in G: g(0)=0\}$ и введем отображение $A: G_{0} \rightarrow C_{0}([0,1], \mathbb{R})=\{u \in$ $\left.C_{0}([0,1], \mathbb{R}): u(0)=u(1)=0\right\}$, полагая $(A g)(\tau)=\left(\frac{g^{\prime \prime}(\tau)}{g^{\prime}(\tau)}\right)^{\prime}-\frac{g^{\prime \prime \prime}(0) g^{\prime}(0)-\left(g^{\prime \prime}(0)\right)^{2}}{\left(g^{\prime}(0)\right)^{2}}$. Пусть $\mu_{\alpha \lambda}^{0}$ - прообраз броуновского моста на $C_{0}([0,1], \mathbb{R})$ с коэффициентом дисперсии $\sqrt{\alpha \lambda}$ относительно отображения $A$.

Как топологическое пространство $G$ представляет собой декартово произведение $S^{1} \times G_{0}$,

$$
g(\tau)=\sigma+g_{0}(\tau) \quad\left(g \in G, \sigma=g(0) \in S^{1}, g_{0} \in G_{0}\right)
$$

Зададим на окружности $S^{1}$ меру Лебега $l_{S}$. В итоге на группе $G$ получим меру $\mu_{\alpha \lambda}=$ $l_{S} \otimes \mu_{\alpha \lambda}^{0}$.

При $\alpha \rightarrow+\infty$ и $\lambda \rightarrow+\infty \mathscr{A}_{1}[\varphi]$ переходит в

$$
\int_{\mathbb{R}^{4}} \int_{\mathbb{R}^{4}} \int_{\mathbb{R}^{4}} \int_{\mathbb{R}^{4}} \delta\left(r_{1}+r_{2}+r_{3}+r_{4}\right) \varphi\left(r_{1}\right) \varphi\left(r_{2}\right) \varphi\left(r_{3}\right) \varphi\left(r_{4}\right) d r_{1} d r_{2} d r_{3} d r_{4}
$$

Полный функционал действия равен

Справедлива следующая теорема.

$$
\mathscr{A}[\varphi]=\mathscr{A}_{0}[\varphi]+\kappa \mathscr{A}_{1}[\varphi] .
$$

ТЕОРема. При произвольном положительном к и любом $\psi \in$ Е существует интег$p a \Omega$

$$
\int_{E} e^{-\mathscr{A}[\varphi]-i(\varphi, \psi)_{E}} d \varphi
$$

ДоказАТЕЛЬСтво. Для любых $\varphi \in E$ функционал $\mathscr{A}_{1}[\varphi]$ принимает неотрицательные значения, поэтому функционал $e^{-\kappa \mathscr{A}_{1}[\varphi]-i(\varphi, \psi)_{E}}$ ограничен на пространстве $E$. Следовательно, для доказательства сходимости интеграла (1) достаточно убедиться в существовании интеграла $\int_{E} \mathscr{A}_{1}[\varphi] e^{-\mathscr{A}_{0}[\varphi]} d \varphi$. Последнее вытекает из следующей серии лемм.

Лемма 1. Существует $c_{1}>0$ такое, ито для любого $r \in \mathbb{R}^{4}, r \neq 0$, выполнено

$$
\|r\|^{2} \int_{\mathscr{P}_{0}}\left(\int_{S^{1}} \frac{d \tau}{\|r+\xi(\tau)\|^{2}}\right) w_{\lambda}^{0}(d \xi) \leqslant c_{1} .
$$

Лемма 2. Существует $c_{2}>0$ такое, что для любих $r_{1}, r_{2} \in \mathbb{R}^{4} u t_{1}, t_{2} \in S^{1}, t_{1} \neq t_{2}$, справедлива оценка

$$
\int_{\mathscr{P}}\left\|p\left(t_{1}\right)\right\|^{2} \delta\left(p\left(t_{1}\right)-r_{1}\right)\left\|p\left(t_{2}\right)\right\|^{2} \delta\left(p\left(t_{2}\right)-r_{2}\right)\left(\int_{S^{1}} \frac{d \tau}{\|p(\tau)\|^{2}}\right)^{2} w_{\lambda}(d p) \leqslant c_{2}
$$

Лемма 3. Существует $c_{3}>0$ такое, ито для любьх $r_{1}, r_{2} \in \mathbb{R}^{4} u t_{1}, t_{2} \in S^{1}, t_{1} \neq t_{2}$, выполнено

$$
\begin{gathered}
\int_{\mathscr{P}}\left[\Omega^{2}\left(p_{1}\right)\right]^{-1} \delta\left(p\left(t_{1}\right)-r_{1}\right)\left\|p\left(t_{1}\right)\right\|^{2} \delta\left(p\left(t_{2}\right)-r_{2}\right)\left\|p\left(t_{2}\right)\right\|^{2} \\
\times\left(\int_{S^{1}} \frac{d \tau}{\|p(\tau)\|^{2}}\right)^{2}\left(\mathcal{U}_{\alpha \lambda}(p)\right)^{2} w_{\lambda}(d p) \leqslant c_{3} .
\end{gathered}
$$

Лемма 4. Существует $c_{4}>0$ такое, что для любъх $r \in \mathbb{R}^{4} u t \in S^{1}$ имеет место оченка

$$
\int_{\mathscr{P}} \delta(p(t)-r)\|p(t)\|^{2} \int_{S^{1}} \frac{d \tau}{\|p(\tau)\|^{2}} \mathscr{U}_{\alpha \lambda}(p) w_{\lambda}(d p) \leqslant c_{4} .
$$

\section{СПИСОК ЛИТЕРАТУРЫ}

[1] E. T. Shavgulidze // Russian J. Math. Phys. 2000. V. 7. № 4. P. 464-472.

Московский государственньй университет им. М.В.Ломоносова E-mail: belokur@rector.msu.ru
Принято редколлегией 23.07 .2004 